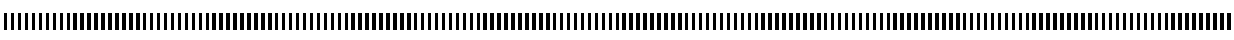

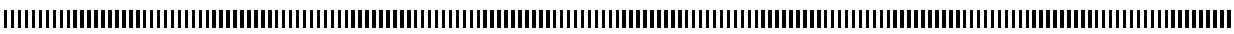

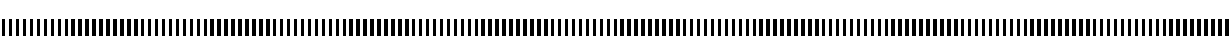

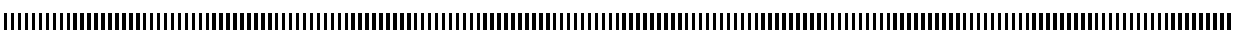

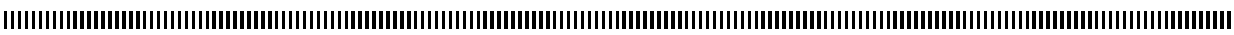

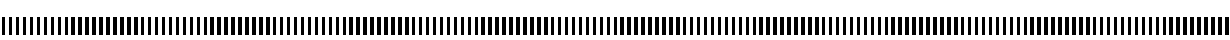

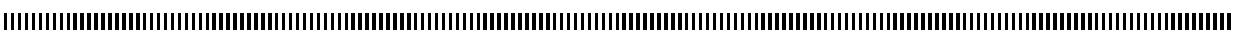

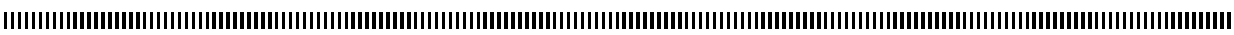

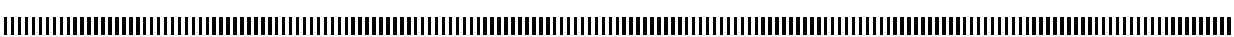

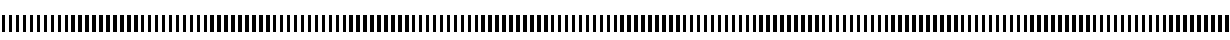

\title{
Schéma SRNHS
}

\section{Analyse et Application d'un schéma aux volumes finis dédié aux systèmes non homogènes}

\author{
Slah Sahmim *, Fayssal Benkhaldoun * \\ * Laboratoire d'Analyse, Geometrie et Applications \\ UMR CNRS 7539 \\ Institut Galilee - Universite Paris Nord \\ Av. J.B. Clement 93430 Villetaneuse.
}

\begin{abstract}
||
RÉSUMÉ. Cet article concerne l'analyse et l'application, d'un schéma proposé récemment pour une classe de systèmes non homogènes. Nous considérons ceux pour lesquels le problème de Riemann correspondant admet une solution autosimilaire. Des exemples importants de tels problèmes sont l'écoulement d'eau peu profonde au-dessus d'un fond non plat et les problèmes diphasiques. L' analyse de stabilité du schéma, dans le cas scalaire homogène, amène à une nouvelle écriture qui a une extension naturelle pour le cas non homogène. Des expériences numériques comparatives pour des équations de Saint-Venant avec topographie variable, et un problème diphasique (Robinet de Ransom) sont présentés pour évaluer l'efficacité du schéma.
\end{abstract}

ABSTRACT. This article is devoted to the analysis, and improvement of a finite volume scheme proposed recently for a class of non homogeneous systems. We consider those for which the corresponding Riemann problem admits a selfsimilar solution. Some important examples of such problems are Shallow Water problems with irregular topography and two phase flows. The stability analysis of the considered scheme, in the homogeneous scalar case, leads to a new formulation which has a natural extension to non homogeneous systems. Comparative numerical experiments for Shallow Water equations with source terms, and a two phase problem (Ransom faucet) are presented to validate the scheme.

MOTS-CLÉS : Méthodes des volumes finis, Equation de Saint-Venant, Terme de source, Problème de Rieman, Problèmes diphasiques, Loi de conservation.

KEYWORDS : Finite volume method, Shallow water equations, Source terms, Riemann Problems, Tow-phase flow, System of conservation laws.

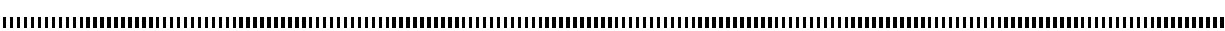

Volume 5 - 2006, pages 302 à 316 - A R I M A

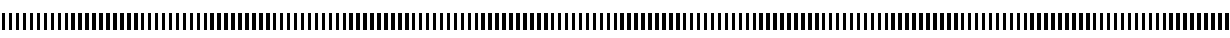

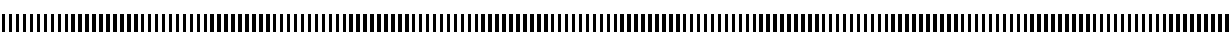

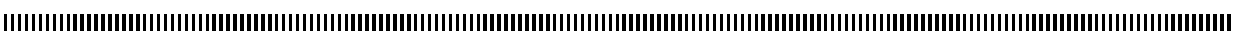

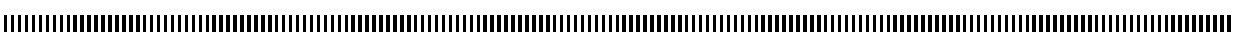

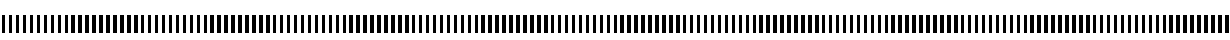
|

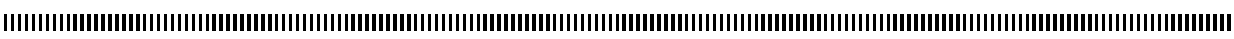
|

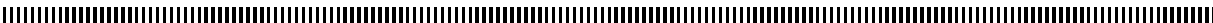




\section{Introduction}

Un grand nombre de problèmes de la mécanique des fluides sont non homogènes ou régis par des équations hyperboliques avec des termes source. Cela est le cas en particulier pour les écoulements en eau peu profnde, régis par les équations de Saint-Venant avec terme source, de même que pour les problèmes diphasiques.

Les équations de Saint-Venant régissent un écoulement à surface libre soumis à la force de gravité. Des problèmes classiques gouvernés par de tels systèmes, sont par exemple les ruptures de barrages, ou les crues. Ces systèmes peuvent poser des grands problèmes de nature numérique en présence de discontinuités de la topographie ou de fond sec.

Un écoulement diphasique est un écoulement constitué d'un mélange de deux phases, comme par exemple la présence de bulles de gaz dans un liquide. Il y a des situations diverses où peuvent apparaitre ces types d'écoulements (cœurs et générateurs de vapeur des réacteurs nucléaires à eau pressurisée, pipelines de transport d'effluents pétroliers, chambre des moteurs à combustion internes, ...). Cette large gamme d'applications conduit à de nombreux modèles rendant chacun compte d'un type de configuration donnée. On peut citer en particulier le modèle diphasique à pression commune, décrit par un système non hyperbolique, et qui pose de grandes difficultés lors de sa simulation numérique par les schémas de volumes finis (VF) décentrés classiques.

Les schémas numériques pour les systèmes non homogènes ou non-conservatifs, ont fait l'objet de plusieurs études ces dernières années. Pour surmonter les difficultés présentées par de tels systèmes, un travail considérable a été réalisé (voir par exemple I. Toumi et A. Kumbaro 1996) [20], et J. M. Ghidaglia [8] pour les écoulements diphasiques à une pression. Pour la simulation numérique des écoulements diphasiques à deux pressions, parmi les méthodes appliquées, on note le schéma VFRoe [19]. Pour les écoulements avec termes sources, on doit mentionner notamment les schémas d'équilibre étudiés par Bermùdez et Vázquez (1994, 1998) [4, 3], Greenberg et Leroux (1996) [9], [6], Leroux [19], et Gosse (1996) [10]. L'idée principale dans cette approche est d'écrire un schéma assurant une représentation précise des états stationnaires. Cela implique un équilibre entre le terme de transport et le terme source, et se traduit par le décentrement de ce dernier.

Précisons que les méthodes citées, se fondent pour l'essentiel sur des solveurs approchés de Riemann [16]. Cela implique la détermination des champs propres d'une Jacobienne associée au système. Cette détermination se fait, dans le cas des systèmes diphasiques par exemple, généralement par le biais de calculs approximatifs plus ou moins ardus, et conduit à varier le traitement en fonction du régime d'écoulement considéré. Cela peut être coûteux mais incontournable pour des écoulements relativement complexes.

Cependant, pour une large gamme de systèmes non homogènes, incluant des écoulements diphasiques, et des écoulements avec termes sources (éventuellement non bornés), on peut 
envisager de développer des schémas n'ayant pas recours aux calculs complexes évoqués plus haut.

Notre but dans ce travail est de considérer une classe particulière de systèmes non conservatifs; à savoir ceux pour lesquels on suppose que la solution du problème associé de Riemann est autosimilaire. Cette classe de problèmes est assez large cependant, et peut contenir divers phénomènes tels que les écoulements diphasiques, ou les écoulements d'eau peu profonde avec topographie irrégulière.

Nous nous basons pour cela sur le schéma Solveur de Riemann Non Homogène (SRNH) proposé par F. Benkhaldoun [2], que nous modifions, en introduisant une vitesse de Rusanov, en vue de son extension au cas bidimensionnel. En effet, comme on le rappelera par la suite, le schéma $(S R N H)$ est constitué de deux étapes, dont la première, que nous avons appelé étape prédicteur, consiste à écrire à l'interface de deux cellules, une approximation décentrée de la solution approché, faisant intervenir un paramètre scalaire de contrôle de la diffusion numérique.

L'analyse de stabilité de ce schéma dans le cas homogène, révèle que le respect de quelques conditions suffisantes pour le principe du maximum amène à réécrire la phase prédicteur du schéma en introduisant le signe d'une matrice, dite de décentrement de l'état. Un nouveau schéma apparaît alors qui s'identifie, dans le cas homogène, au schéma VFRoe introduit par T. Gallouët [13], mais qui a ici une extension naturelle pour le cas non homogène et se révèlera efficace aussi bien pour des problèmes d'écoulement d'eau que pour des problèmes diphasiques.

Le présent article est structuré de la manière suivante : En section 2, nous rappelons l'écriture du schéma $S R N H$, et introduisons la vitesse de Rusanov. La section 3, corps de l'article, est dédiée à l'analyse du schéma dans le cas scalaire homogène, et à l'introduction de la matrice signe dans une nouvelle version du schéma. En section 4, nous appliquons le schéma aux écoulement d'eau peu profonde sur fond irrégulier, et avant de conclure nous appliquons, en section 5, le schéma à un écoulement diphasique.

\section{Un schéma proposé pour les problèmes non homogènes : le schéma SRNH}

Considérons un système de lois de bilan dans $\mathbb{R}^{m}$, issue de la mécanique des fluides, et qui s'écrit :

$$
\left\{\begin{array}{l}
\left.\frac{\partial W}{\partial t}+\frac{\partial F(W)}{\partial x}=Q(x, W)=H(W) \frac{\partial G(x, W)}{\partial x} \text { dans } \mathbb{R} \times\right] 0, T[ \\
W(x, 0)=W_{0}(x),
\end{array}\right.
$$


on suppose de plus que le problème de Riemann correspondant: $W_{0}(x)=W_{L}$ si $x<0$, et $W_{0}(x)=W_{R}$ si $x>0$, admet une solution autosimilaire : $W(x, t)=R s\left(\frac{x}{t}, W_{L}, W_{R}\right)$. Dans [2], en se basant sur la propriété de l'autosimilarité de la solution du problème de Riemann, F. Benkhaldoun a déduit un schéma de volumes finis pour les systèmes non homogène en fonction d'un paramètre de contrôle $\theta=\frac{\alpha_{i+\frac{1}{2}}^{n}}{2} \Delta t$ et qui s'écrit en deux étape de la manière suivante :

$$
\left\{\begin{array}{l}
W_{i+\frac{1}{2}}^{n}=\frac{1}{2}\left(W_{i}^{n}+W_{i+1}^{n}\right)-\frac{\alpha_{i+\frac{1}{2}}^{n}}{2} r\left[F\left(W_{i+1}^{n}\right)-F\left(W_{i}^{n}\right)\right]+\frac{\alpha_{i+\frac{1}{2}}^{n}}{2} \Delta t Q_{i+\frac{1}{2}}^{n} \\
W_{i}^{n+1}=W_{i}^{n}-r\left[F\left(W_{i+\frac{1}{2}}^{n}\right)-F\left(W_{i-\frac{1}{2}}^{n}\right)\right]+\Delta t Q_{i}^{n},
\end{array}\right.
$$

où $r=\frac{\Delta t}{\Delta x}$, avec $\Delta t$ le pas de temps et $\Delta x$ le pas d'espace.

$$
Q_{i+\frac{1}{2}}^{n}=\frac{E\left(W_{i}^{n}\right)+E\left(W_{i+1}^{n}\right)}{2} \frac{G\left(x_{i+1}, W_{i+1}^{n}\right)-G\left(x_{i}, W_{i}^{n}\right)}{\Delta x} \quad \text { et } \quad Q_{i}^{n}
$$

sont respectivement les approximations du terme source dans l'étape prédicteur et l'étape correcteur.

Dans le but de faire l'extension du schéma $S R N H$ au cas bidimensionnel, où il est difficile de définir un équivalent de la distance $\Delta x$, il est apparu nécessaire de proposer une variante de $(S R N H)$ ne faisant pas apparaître cette distance dans la phase prédicteur du schéma $[17,18]$.

Cela consiste à écrire $\theta=\alpha_{i+\frac{1}{2}}^{n} \bar{\theta}$ où $\bar{\theta}$ est défini par la vitesse de Rusanov comme suit :

$$
\bar{\theta}=\frac{\Delta x}{2 s_{i+\frac{1}{2}}^{n}} \quad \text { et } \quad s_{i+\frac{1}{2}}^{n}=\max _{p=1, \ldots, m}\left(\max \left(\left|\lambda_{p, i}^{n}\right|,\left|\lambda_{p, i+1}^{n}\right|\right)\right)
$$

Ceci nous amène à la nouvelle expression du schéma :

$$
\left\{\begin{array}{l}
W_{i+\frac{1}{2}}^{n}=\frac{1}{2}\left(W_{i}^{n}+W_{i+1}^{n}\right)-\frac{\alpha_{i+\frac{1}{2}}^{n}}{2 s_{i+\frac{1}{2}}^{n}}\left[F\left(W_{i+1}^{n}\right)-F\left(W_{i}^{n}\right)\right]+\frac{\alpha_{i+\frac{1}{2}}^{n}}{2} \frac{\Delta x}{s_{i+\frac{1}{2}}^{n}} Q_{i+\frac{1}{2}}^{n} \\
W_{i}^{n+1}=W_{i}^{n}-r\left[F\left(W_{i+\frac{1}{2}}^{n}\right)-F\left(W_{i-\frac{1}{2}}^{n}\right)\right]+\Delta t Q_{i}^{n} .
\end{array}\right.
$$




\section{Analyse du schéma et introduction de la matrice signe}

\subsection{Cas scalaire}

Soit la fonction flux $f: \mathbb{R} \rightarrow \mathbb{R}$ une fonction de classe $\mathcal{C}^{1}$ non linéaire, $w_{0} \in L^{\infty}(\mathbb{R})$ étant la condition initiale du problème (1).

Pour $\gamma \geq 1$, On définit l'ensemble suivant :

$$
X=\left\{w \in \mathbb{R} /|w| \leq \gamma|| w_{0} \|_{L^{\infty}(\mathbb{R})}\right\}
$$

et on pose $A=\max _{w \in X}\left|f^{\prime}(w)\right|$.

Le schéma de volumes finis (2) est conservatif, consistant par construction et la fonction du flux numérique est donnée par $g\left(w_{i}^{n}, w_{i+1}^{n}\right)=f\left(w_{i+\frac{1}{2}}^{n}\right)$. On note $a \perp b=\min (a, b)$ et $a \top b=\max (a, b)$.

Proposition 3.1 On suppose que $f$ est une fonction de classe $C^{1}$, monotone et que la partie non homogène est nulle, alors sous les deux conditions suivantes :

1) $\frac{s_{i+\frac{1}{2}}^{n}}{\left|f^{\prime}\left(a_{i+\frac{1}{2}}^{n}\right)\right|} \leq \alpha_{i+\frac{1}{2}}^{n} \leq \gamma \frac{s_{i+\frac{1}{2}}^{n}}{\left|f^{\prime}\left(a_{i+\frac{1}{2}}^{n}\right)\right|}, \forall i \in \mathbb{Z}, n \in \mathbb{N}$,

2) $r \leq \frac{1}{\gamma A}$

avec

$$
a_{i+\frac{1}{2}}^{n} \in\left[w_{i+1}^{n} \perp w_{i}^{n}, w_{i+1}^{n} \top w_{i}^{n}\right]
$$

vérifiant

$$
f\left(w_{i+1}^{n}\right)-f\left(w_{i}^{n}\right)=f^{\prime}\left(a_{i+\frac{1}{2}}^{n}\right)\left(w_{i+1}^{n}-w_{i}^{n}\right),
$$

le schéma SRNH satisfait au principe du maximum local :

$$
\min _{i \in \mathbb{Z}} w_{i}^{n} \leq \min _{i \in \mathbb{Z}} w_{i}^{n+1} \leq \max _{i \in \mathbb{Z}} w_{i}^{n+1} \leq \max _{i \in \mathbb{Z}} w_{i}^{n} .
$$

Preuve $f$ étant continue et dérivable, le théorème des accroissements finis, nous permet d'écrire qu'il existe $a_{i}^{n}$ et $a_{i+\frac{1}{2}}^{n}$ tels que

$$
w_{i+\frac{1}{2}}^{n}=\frac{1}{2}\left(1+\delta_{i+\frac{1}{2}}^{n}\right) w_{i}^{n}+\frac{1}{2}\left(1-\delta_{i+\frac{1}{2}}^{n}\right) w_{i+1}^{n} \quad \text { avec } \quad \delta_{i+\frac{1}{2}}^{n}=\frac{\alpha_{i+\frac{1}{2}}^{n}}{S_{i+\frac{1}{2}}^{n}} f^{\prime}\left(a_{i+\frac{1}{2}}^{n}\right),
$$

et

$$
w_{i}^{n+1}=w_{i}^{n}-r f^{\prime}\left(a_{i}^{n}\right)\left(w_{i+\frac{1}{2}}^{n}-w_{i-\frac{1}{2}}^{n}\right)
$$


où $f^{\prime}\left(a_{i}^{n}\right)\left(w_{i+\frac{1}{2}}^{n}-w_{i-\frac{1}{2}}^{n}\right)=f\left(w_{i+\frac{1}{2}}^{n}\right)-f\left(w_{i-\frac{1}{2}}^{n}\right)$

Sous les deux conditions de la proposition (3.1), $w_{i}^{n+1}$ s'écrit comme une combinaison convexe en fonction de $w_{i-1}^{n}, w_{i}^{n}$ et $w_{i+1}^{n}$, il s'ensuit le résultat du principe de maximum local et la stabilité $L^{\infty}$.

\subsection{Le paramètre de contrôle dans l'étape prédicteur}

La condition de stabilité 2) de la proposition (3.1) exige que $\left|f^{\prime}\left(a_{i}^{n}\right)\right|$ soit finie, une condition suffisante pour cela, est que $a_{i}^{n}$ et $w_{i+\frac{1}{2}}^{n}$ restent bornés.

Dans ce qui précède, cela a été obtenu, en imposant que $w_{i+\frac{1}{2}}^{n} \in X$, où $X$ est défini au début de cette section via un paramètre $\gamma \geq 1$. Nous voulons ici lever l'indétermination introduite par ce paramètre $\gamma$. Pour cela, on remarque qu'une condition suffisante pour que $w_{i+\frac{1}{2}}^{n}$ reste borné est que

$$
w_{i+\frac{1}{2}}^{n} \in\left[w_{i}^{n} \perp w_{i+1}^{n}, w_{i}^{n} \top w_{i+1}^{n}\right] .
$$

Cela implique un choix unique possible pour $\alpha_{i+\frac{1}{2}}^{n}$, qui est $\alpha_{i+\frac{1}{2}}^{n}=\frac{S_{i+\frac{1}{2}}^{n}}{\left|f^{\prime}\left(a_{i+\frac{1}{2}}^{n}\right)\right|}$ [18].

Finalement on déduit le schéma $S R N H S$ (c'est le schéma $S R N H$ en fixant le paramètre de contrôle $\alpha_{i+\frac{1}{2}}^{n}$ en fonction du signe $f^{\prime}$ dans l'étape prédicteur), qui s'écrit sous la forme :

$$
\left\{\begin{array}{l}
w_{i+\frac{1}{2}}^{n}=\frac{1}{2}\left(w_{i}^{n}+w_{i+1}^{n}\right)-\frac{1}{2\left|f^{\prime}\left(a_{i+\frac{1}{2}}^{n}\right)\right|}\left(f\left(w_{i+1}^{n}\right)-f\left(w_{i}^{n}\right)\right)+\frac{\Delta x}{2\left|f^{\prime}\left(a_{i+\frac{1}{2}}^{n}\right)\right|} Q_{i+\frac{1}{2}}^{n} \\
w_{i}^{n+1}=w_{i}^{n}-r\left(f\left(w_{i+\frac{1}{2}}^{n}\right)-f\left(w_{i-\frac{1}{2}}^{n}\right)\right)+\Delta t Q_{i}^{n},
\end{array}\right.
$$

soit

$$
\left\{\begin{array}{l}
w_{i+\frac{1}{2}}^{n}=\frac{1}{2}\left(w_{i}^{n}+w_{i+1}^{n}\right)-\frac{1}{2} \operatorname{sgn}\left(f^{\prime}\left(a_{i+\frac{1}{2}}^{n}\right)\right)\left(w_{i+1}^{n}-w_{i}^{n}\right)+\frac{\Delta x}{2\left|f^{\prime}\left(a_{i+\frac{1}{2}}^{n}\right)\right|} Q_{i+\frac{1}{2}}^{n} \\
w_{i}^{n+1}=w_{i}^{n}-r\left(f\left(w_{i+\frac{1}{2}}^{n}\right)-f\left(w_{i-\frac{1}{2}}^{n}\right)\right)+\Delta t Q_{i}^{n} .
\end{array}\right.
$$

\subsection{Cas de systèmes homogènes}

On considère le système hyperbolique linéaire homogène $1 D$ suivant :

$$
\left\{\begin{array}{l}
\frac{\partial W}{\partial t}+\mathcal{A} \frac{\partial W}{\partial x}=0,(x, t) \in \mathcal{D} \times \mathbb{R}_{+}^{*}, \mathcal{D} \subset \mathbb{R} \\
W(x, 0)=W_{0}(x), x \in \mathcal{D} .
\end{array}\right.
$$


$\mathcal{A}$ étant diagonalisable dans $\mathbb{R}$, on a $\mathcal{A}=\mathcal{R} \Lambda \mathcal{R}^{-1}$. En injectant cette expression dans le schéma (2), on aboutit à un schéma équivalent exprimé sur la variable caractéristique $V=\mathcal{R}^{-1} W$, et se déclinant en $m$ équations sur les composantes de $V$, sous la forme :

$$
\left\{\begin{array}{l}
\left(v_{p}\right)_{i+\frac{1}{2}}^{n}=\frac{1}{2}\left(\left(v_{p}\right)_{i+1}^{n}+\left(v_{p}\right)_{i}^{n}\right)-\frac{\alpha_{i+\frac{1}{2}}^{n}}{2 s_{i+\frac{1}{2}}^{n}} \lambda_{p}\left(\left(v_{p}\right)_{i+1}^{n}-\left(v_{p}\right)_{i}^{n}\right) \\
\left(v_{p}\right)_{i}^{n+1}=\left(v_{p}\right)_{i}^{n}-r \lambda_{p}\left(\left(v_{p}\right)_{i+\frac{1}{2}}^{n}-\left(v_{p}\right)_{i-\frac{1}{2}}^{n}\right)
\end{array}\right.
$$

L'analyse de stabilité et le principe du maximum pour le schéma scalaire (proposition 3.1 ), montre qu'il suffit de choisir le paramètre de contrôle sous forme matricielle $\alpha_{i+\frac{1}{2}}^{n}=$ $s_{i+\frac{1}{2}}^{n} \operatorname{diag}\left(\frac{1}{\left|\lambda_{k}\right|}\right)$, afin d'assurer la condition (3), composante par composante. En injectant cette expression dans le schéma (2), on obtient le schéma SRNHS

$$
\left\{\begin{array}{l}
W_{i+\frac{1}{2}}^{n}=\frac{1}{2}\left(W_{i+1}^{n}+W_{i}^{n}\right)-\frac{1}{2} \operatorname{sgn}(\mathcal{A})\left(W_{i+1}^{n}-W_{i}^{n}\right) \\
W_{i}^{n+1}=W_{i}^{n}-r\left(F\left(W_{i+\frac{1}{2}}^{n}\right)-F\left(W_{i-\frac{1}{2}}^{n}\right)\right)
\end{array}\right.
$$

$\operatorname{avec} \operatorname{sgn}(\mathcal{A})=\mathcal{R} \operatorname{sgn}(\Lambda) \mathcal{R}^{-1}$ et $\operatorname{sgn}(\Lambda)=\operatorname{diag}\left(\frac{\lambda_{k}}{\left|\lambda_{k}\right|}\right)$.

Pour les problèmes non linéaires avec terme source on applique la linéarisation locale de Roe pour le schéma au niveau de l'étape prédicteur, le schéma obtenu s'écrit alors :

$$
\left\{\begin{array}{l}
W_{i+\frac{1}{2}}^{n}=\frac{1}{2}\left(W_{i+1}^{n}+W_{i}^{n}\right)-\frac{1}{2} \operatorname{sgn}(\nabla F(\bar{W}))\left(W_{i+1}^{n}-W_{i}^{n}\right)+\frac{1}{2}|\nabla F(\bar{W})|^{-1} \Delta x Q_{i+\frac{1}{2}}^{n} \\
W_{i}^{n+1}=W_{i}^{n}-r\left(F\left(W_{i+\frac{1}{2}}^{n}\right)-F\left(W_{i-\frac{1}{2}}^{n}\right)\right)+\Delta t Q_{i}^{n}
\end{array}\right.
$$

où $\nabla F(\bar{W})$ est la Jacobienne de $F$ calculée à l'état moyen de Roe $\bar{W}=\bar{W}\left(W_{i}^{n}, W_{i+1}^{n}\right)$ telque $F\left(W_{i+1}^{n}\right)-F\left(W_{i}^{n}\right)=\nabla F(\bar{W})\left(W_{i+1}^{n}-W_{i}^{n}\right)$.

Remarque 3.2 Pour tous les tests numérique, nous avons constaté que les résultats sont identiques que l'on prenne l'état de Roe, ou juste la moyenne arithmétique : $\bar{W}=\frac{1}{2}\left(W_{i}^{n}+W_{i+1}^{n}\right)$. 


\section{Application du schéma SRNHS pour le problème de Saint-Venant avec topographie irrégulière}

\subsection{Description de la méthode}

Nous allons utiliser le schéma $S R N H S$ pour la simulation numérique du problème de Saint-Venant avec un fond irrégulier $z$ dépendant seulement de la variable spatiale $x$. $h(x, t)$ représente la hauteur de l'eau, $\zeta(x, t)=h(x, t)+z(x)$ la surface libre, $u(x, t)$ la vitesse, et $q(x, t)=h(x, t) u(x, t)$ le débit. L'équation de Saint-Venant s'écrit dans ce cas sous la forme suivante :

$$
\left\{\begin{array}{l}
\frac{\partial W}{\partial t}+\frac{\partial F(W)}{\partial x}=Q(x, w) \\
W(x, 0)=W_{0}(x)
\end{array}\right.
$$

$$
\begin{aligned}
& \text { avec } W(x, t)=\left(\begin{array}{c}
h(x, t) \\
h u(x, t)
\end{array}\right), \quad F(W(x, t))=\left(\begin{array}{c}
h u(x, t) \\
h u^{2}(x, t)+\frac{g}{2} h^{2}
\end{array}\right) \\
& \text { et } Q(x, t)=\left(\begin{array}{c}
0 \\
-g h \frac{d z(x)}{d}
\end{array}\right) .
\end{aligned}
$$

\section{Conservation de l'état d'équilibre}

Soit $W(x, t)$ une solution stationnaire de (6), c'est-à-dire $W$ est assez régulière vérifiant $\frac{\partial W}{\partial t}(x, t)=0$ et $u(x, t)=0$. Dans ce cas la deuxième équation du système (6) est équivalente à :

$$
\frac{1}{2} \frac{\partial}{\partial x}\left(g h^{2}\right)(x, t)=-g h \frac{\partial z}{\partial x}(x) \quad \Longleftrightarrow \quad h(x, t)+z(x)=c .
$$

Définition 4.1 On dit que le schéma vérifie la $\mathcal{C}$-propriété $[4,3]$, si le schéma appliqué à une solution stationnaire retrouve

$$
h_{i}^{n}+z_{i}=c, \quad \text { et } \quad u_{i}^{n}=0 \quad \forall i \in \mathbb{Z}, \forall n \in \mathbb{N} .
$$

Proposition 4.2 Sous la condition de la discrétisation de la deuxième composante du terme source dans l'étape correcteur sous l'une des deux formes suivantes :

i) $\left(Q_{i}^{n}\right)_{2}=-\frac{g}{4 \Delta x}\left(h_{i+\frac{1}{2}}^{n}+h_{i-\frac{1}{2}}^{n}\right)\left(z_{i+1}-z_{i-1}\right)$, 
ii) $\left(Q_{i}^{n}\right)_{2}=-\frac{g}{8 \Delta x}\left(h_{i+1}^{n}+2 h_{i}^{n}+h_{i-1}^{n}\right)\left(z_{i+1}-z_{i-1}\right)$,

le schéma $S R N H S$ vérifie la $\mathcal{C}$-propriété.

La preuve est faite dans [18].

\subsection{Ecoulement d'eau sur un fond discontinu}

\subsubsection{Rupture de barrage sur une marche avec transistion critique}

Il s'agit d'un cas test avec double singularité [1] : une singularité au niveau des conditions initiales, plus la discontinuité du fond.

On prend comme condition initiale :

$$
h_{l}=5 m \quad h_{r}=1, \quad u_{l}=-4 m / s, \quad u_{r}=9 m / s .
$$

Le nombre de Froude $\left(F r=\frac{u}{\sqrt{g h}}\right)$ atteint la valeur critique 1 au niveau de la marche. C'est un cas difficile qui a nécessité le passage à l'ordre deux en espace par la méthode $M U S C L$ [11] afin d'obtenir une solution correcte et de garande précision (voir FIG. 1). Toutefois, nous remarquons au travers de la courbe de convergence (voir FIG. 2), que même à l'ordre 1, le schéma $S R N H S$ a un bon comportement, comparé au désormais bien connu schéma équilibre de Bermudez et Vazquez ([3].

\subsubsection{Ecoulement d'eau sur un obstacle, avec apparition de fond sec}

Ce cas est traité en particulier dans [19] par le schéma VFRoe étendu au cas non homogène. Initialement nous avons un surface libre constante, égale à 10 , et une condition de Riemann sur le débit, avec pour valeurs -350 et +350 de part et d'autre du point $x=17$. Nous obtenons des résultas identiques à ceux de [19]. En particulier l'apparition du fond sec, et sa progression sur l'obstacle, sont remarquablement bien représentés (voir Fig. 3).

\section{Application du schéma SRNHS au problème de Ransom}

\subsection{Modèle bi-fluide}

Dans cette partie nous considérons un modèle bi-fluide à quatre équations à une pression commune, dont deux équations décrivent la conservation de la masse, et deux équations 
décrivent la conservation du moment des deux phases. Dans ce cas le système avec condition initiale s'écrit de la manière suivante :

$$
\left\{\begin{array}{l}
\frac{\partial W(x, t)}{\partial t}+\frac{\partial F(W(x, t))}{\partial x}+S_{1}(x, W)=S_{2}(x, W) \\
W(x, 0)=W_{0}(x)
\end{array}\right.
$$

avec

$$
\begin{gathered}
W(x, t)=\left(\begin{array}{c}
\alpha_{v} \rho_{v} \\
\alpha_{v} \rho_{v} u_{v} \\
\alpha_{l} \rho_{l} \\
\alpha_{l} \rho_{l} u_{l}
\end{array}\right), \quad F(W(x, t))=\left(\begin{array}{c}
\alpha_{v} \rho_{v} u_{v} \\
\alpha_{v} \rho_{v} u_{v}^{2} \\
\alpha_{l} \rho_{l} u_{l} \\
\alpha_{l} \rho_{l} u_{l}^{2}
\end{array}\right), \\
0 \\
S_{1}(x, W)=\left(\begin{array}{c}
\alpha_{v} \frac{\partial p}{\partial x}+\left(p-p_{v}^{i}\right) \frac{\partial \alpha_{v}}{\partial x} \\
0 \\
\alpha_{l} \frac{\partial p}{\partial x}+\left(p-p_{l}^{i}\right) \frac{\partial \alpha_{l}}{\partial x}
\end{array}\right), \quad \text { et } S_{2}(x, W)=\left(\begin{array}{c}
0 \\
\alpha_{v} \rho_{v} g \\
0 \\
\alpha_{l} \rho_{l} g
\end{array}\right),
\end{gathered}
$$

où : $v$ et $l$ se rapportent aux phases vapeur et liquides respectivement, $g$ est l'accélération de la pesanteur, $\alpha, \rho$ et $u$ sont respectivement le taux de présence, la densité et la vitesse de la phase $k(k=v$ ou $l)$. On a sept inconnues $\left(\alpha_{v}, \alpha_{l}, \rho_{v}, \rho_{l}, u_{v}, u_{l}, p\right)$ et quatre équations, donc il nous faut des lois d'états pour fermer le système (7). La première loi lie les taux de vide : $\alpha_{v}+\alpha_{l}=1$, et la deuxième celle des gaz parfait, gouverne la pression en fonction des densités de la vapeur et du liquide $: p=c \rho_{v}^{\gamma}$ et $\rho_{l}=k_{l} p^{a}$ où $c, \gamma, a$ et $k_{l}$ sont des constantes données par $c=10^{5}, \gamma=1.4, a=4.37 \times 10^{-5}$ et $k_{l}=987,57$ (Système S.I). Pour des raisons de simplicité on suppose que la densité du liquide est quasi-constante $\rho_{l} \simeq \rho_{l}^{0}$ et que la phase vapeur est très légère par rapport à la phase liquide. $p_{k}^{i}$ est la pression interfaciale des deux phases, le terme différentiel $\left(p-p_{k}^{i}\right) \frac{\partial \alpha_{k}}{\partial x}$ est le terme de pression de correction. Il y a plusieurs variétés de l'expression $\left(p-p_{k}^{i}\right)[15,5]$. Ici nous adoptons la loi de fermeture des flux de bulles présenté par R.T. Lahaye [14] qui définit $p-p_{k}^{i}$ comme suit

$$
\left\{\begin{array}{l}
p-p_{v}^{i}=0 \\
p-p_{l}^{i}=C_{p}\left(\alpha_{v}\right) \rho_{l}\left(u_{v}-u_{l}\right)^{2}
\end{array}\right.
$$

avec $C_{p}\left(\alpha_{v}\right)=\delta \alpha_{v}$ ou $C_{p}\left(\alpha_{v}\right)=\frac{\delta \alpha_{v} \alpha_{l} \rho_{v}}{\alpha_{v} \rho_{l}+\alpha_{l} \rho_{l}}$, où $\delta$ est une constante choisie.

Dans la suite on pose $\theta_{l}=p-p_{l}^{i}$.

\subsection{Méthode de la perturbation par densités}

Dans la première partie de ce travail une analyse mathématique du schéma $S R N H$ [2] pour les systèmes hyperboliques non homogènes a abouti à un nouveau schéma de type prédicteur-correcteur appelé SRNHS défini par (5). 
Ici, dans la mesure où le système bifluide n'est pas hyperbolique, $\nabla F(W)$ n'est pas diagonalisable en général. Cependant, la jacobienne $\nabla F(W)$ n'ayant pour rôle dans notre schéma, que de décentrer l'état approché intermédiaire dans l'étape prédicteur, nous pouvons nous contenter d'une jacobienne "proche", qui soit diagonalisable dans $\mathbb{R}$. Pour cela on écrit le système (7) sous la forme suivante :

$$
\frac{\partial W(x, t)}{\partial t}+\nabla F(W(x, t)) \frac{\partial W(x, t)}{\partial x}+C(W) \frac{\partial W(x, t)}{\partial x}=S_{2}(x, W),
$$

avec $C(W) \frac{\partial W(x, t)}{\partial x}=S_{1}(x, W)$. Ensuite on utilise le schéma de fractionnement en deux étapes. Dans la première étape on utilise le schéma d'Euler d'integration en temps

$$
\left\{\begin{array}{l}
\frac{\partial W}{\partial t}=S_{2}(\hat{W}) \\
\hat{W}\left(x, t^{n}\right)=W^{n}(x)
\end{array}\right.
$$

ensuite avec le schéma $S R N H S$ on résout le système

$$
\left\{\begin{array}{l}
\frac{\partial W(x, t)}{\partial t}+\frac{\partial F(W(x, t))}{\partial x}+S_{1}(x, W)=0 \\
W\left(x, t^{n}\right)=W^{n+1}(x) .
\end{array}\right.
$$

Ainsi (8) équivaut à

$$
\frac{\partial W(x, t)}{\partial t}+(\nabla F(W(x, t))+C(W)) \frac{\partial W(x, t)}{\partial x}=0 .
$$

On pose $\mathcal{A}(W)=\nabla F(W(x, t))+C(W)$.

L'étape prédicteur revient à approcher la solution exacte du problème de Riemann suivant :

$$
\left\{\begin{array}{l}
\frac{\partial W(x, t)}{\partial t}+\mathcal{A}(W) \frac{\partial W(x, t)}{\partial x}=0 \\
W(x, 0)=W_{0}(x) .
\end{array}\right.
$$

En conservant les notations de la première partie, cela revient à écrire :

$$
W_{i+\frac{1}{2}}^{n}=\frac{1}{2}\left(W_{i}^{n}+W_{i+1}^{n}\right)-\frac{1}{2} \operatorname{sgn}(\mathcal{A}(\bar{W}))\left(W_{i+1}^{n}-W_{i}^{n}\right) .
$$

On applique alors la méthode de perturbation par les densités proposées par J. Cortes , A. Debussche et I. Toumi [7], en effectuant le changement de variable suivant $\tilde{\rho_{v}}=\frac{\rho_{v}}{\rho_{v}^{0}}$ et $\tilde{\rho}_{l}=\frac{\rho_{l}}{\rho_{l}^{0}}$ où $\rho_{v}^{0}$ et $\rho_{l}^{0}$ sont deux densités caractéristiques respectivement de la vapeur et du liquide. Ensuite on définit $\epsilon=\frac{\rho_{v}^{0}}{\rho_{l}^{0}}$. Comme on a supposé que la phase vapeur est très 
légère par rapport à la phase liquide, alors $\epsilon<<1$. En tenant compte du calcul fait dans [8] dans le cas où le fluide liquide est incompressible, on a alors :

$$
\mathcal{A}(W)=\mathcal{A}_{0}(W)+\epsilon \mathcal{H}(W)
$$

avec

$$
\mathcal{A}_{0}(W)=\left(\begin{array}{cccc}
0 & 1 & 0 & 0 \\
-u_{v}^{2}+c_{1}^{2} & 2 u_{v} & 0 & 0 \\
0 & 0 & 0 & 1 \\
0 & 0 & -u_{l}^{2}+\frac{\theta_{l}}{\rho_{l}} & 2 u_{l}
\end{array}\right)
$$

et

$$
\mathcal{H}(W)=\left(\begin{array}{cccc}
0 & 0 & 0 & 0 \\
0 & 0 & \frac{\gamma p}{\rho_{v}^{0}} & 0 \\
0 & 0 & 0 & 0 \\
\alpha_{l} p_{, 1} & 0 & \alpha_{l} p_{, 3} & 0
\end{array}\right)
$$

on pose $c_{1}^{2}=\frac{\gamma p}{\rho_{v}}$ et $c_{2}^{2}=\frac{\theta_{l}}{\rho_{l}}$.

On montre alors que $\mathcal{A}_{0}$ admet quatre valeurs propres distinctes :

$$
\lambda_{1}=u_{v}-c_{1}, \quad \lambda_{2}=u_{v}+c_{1}, \quad \lambda_{3}=u_{l}-c_{2}, \quad \text { et } \quad \lambda_{4}=u_{l}+c_{2}
$$

Comme $\epsilon<<1$, la théorie des perturbations des opérateurs linéaires [12], nous permet alors de traiter le système par le signe de la matrice $\mathcal{A}_{0}$ au lieu du signe de la matrice $\mathcal{A}$. Finalement le schéma $S R N H S$ s'ecrit pour le problème bifluide (7) de la manière suivante :

$$
\left\{\begin{array}{l}
W_{i+\frac{1}{2}}^{n}=\frac{1}{2}\left(W_{i}^{n}+W_{i+1}^{n}\right)-\frac{1}{2} \operatorname{sgn}\left(\mathcal{A}_{0}(\bar{W})\right)\left(W_{i+1}^{n}-W_{i}^{n}\right) \\
W_{i}^{n+1}=W_{i}^{n}-r\left(F\left(W_{i+\frac{1}{2}}^{n}\right)^{-}-F\left(W_{i-\frac{1}{2}}^{n}\right)\right)+\Delta t S_{i}^{n} .
\end{array}\right.
$$

\subsection{Application Numérique - Robinet de Ransom}

Il s'agit d'un cas test monodimensionnel instationnaire proposé par V.H. Ransom comme "benchmark" numérique pour les codes de thermohydrolique diphasique $1 D$ [15].

On considère l'écoulement d'une colonne d'eau à la sortie d'un robinet débouchant dans une enceinte contenant de l'air. La vitesse d'entrée de l'eau est de $10 \mathrm{~m} / \mathrm{s}$, alors que l'air est au repos. Le rapport des sections de la buse de l'enceinte est tel que le taux de vide integré sur la section vaut 0.2 . Dans cette configuration, on observe un phénomène de striction du jet sous l'effet de la pesanteur. En effet, si on fait l'hypothèse que le jet reste cohérant (pas d'arrachage du liquide sous forme de gouttes, pas de pénétration de l'air dans le jet), l'accélération du liquide due à la pesanteur entraîne nécessairement un rétrécissement de la section de passage du liquide par conservation du débit. La configuration 
initiale étant donnée par la solution que l'on obtiendrait en l'absence de pesanteur (donc $\alpha=0.2$ partout), une onde de taux de vide doit se propager de la section d'entrée vers la section de sortie à partir de l'instant initial.

Les conditions aux limites sont implementées de la façon suivante :

à l'entrée on impose sur les variables $\alpha_{v}(0, t)=0.2, u_{v}(0, t)=0, u_{l}(0, t)=10$ avec sortie libre. Tandis que pour la pression on impose une sortie $p(12, t)=10^{5}$ avec entrée libre.

Les solutions numériques sont comparées à la solution exacte au temsp $t=0.6 \mathrm{~s}$, d'un problème bien posé, obtenu en admettant les densités constantes. Nous notons que le schéma $S R N H S$ produit un résultat convenable qualitativement, avec seulement 48 points de maillage. A titre de comparaison, le schéma Lax-Friedrich requiert 2000 points pour un résultat similaire. En outre le scéma $S R N H S$ supporte un raffinement de maillage à hauteur de 600 points, ce qui dénote une bonne robustesse.

\section{Conclusion}

Dans ce travail, nous avons proposé un schéma aux volumes finis $S R N H S$, dédié aux systèmes non homogènes. Le point fondamental de cette approche consiste à utiliser une étape de décentrement de l'état et non du flux. Ce décentrement, qui concerne à la fois les parties homogènes et non homogènes du systèmes, se fait via l'introduction du signe d'une matrice. Dans les cas complexes, ou non hyperboliques, une approximée de la jacobienne du système est suffisante pour opérer le décentrement. Cela donne finalement un schéma de coût très réduit, et dont les performances sont au moins comparables au schémas classiquement utilisés pour ce type de problèmes. Nous nous proposons, en prolongement de ce travail, de faire l'extension de ce schéma aux écoulements diphasiques multidimensionnels.

\section{Bibliographie}

[1] Alcrudo F., Benkhaldoun F., « Exact solutions to the Riemann problem of the shallow water equations with a bottom step », Computers \& Fluids. An International Journal, vol. 30, $\mathrm{n}^{\circ}$ 6, 2001, p. 643-671.

[2] Benkhaldoun, F., "Analysis and validation of a new finite volume scheme for nonhomogeneous systems ", FVCA3, HPS, R. Herbin, D. Kröner(Eds), Proceedings of the Third International Symposium on Finite Volumes for Complex Application, 2002, p. 229-245.

[3] Bermúdez, A., Dervieux, A., Desideri, J.A, Vasquez, M.E., « Upwind schemes for the two-dimensional shallow water equations with variable depth using unstructured meshes », Computers Methods in Applied Mechanics and Engineering , vol. 155, $\mathrm{n}^{\circ}$ 1-2, 1998, p. 48-72. 
[4] Bermúdez, A., VÁsquez M.E., « Upwind methods for hyperbolic conservation laws with source terms », Computers \& Fluids. An International Journal, vol. 23, n ${ }^{\circ} 1-2,1994$, p. 1049 1071.

[5] Bestion, D., « The physical closure laws in the cathare code », Nuclear Engineering and Design, vol. 124, 1999, p. 429-445.

[6] Chinnayya, A., Le Roux, A.Y, Seguin, N. " A well balanced numerical scheme for the approximation of the shallow water equations with topography : the resonant phenomena », Int. J. Finite Volumes. http ://averoes.math.univ-paris13.fr vol. 1, $\mathrm{n}^{\circ}$ 1, 2004.

[7] Cortez J., Debusshe A., Toumi I. « A density perturbation method to study the eigenstructure of two-phase flow equation systems », Journal of Computational Physics, vol. 147, $\mathrm{n}^{\circ} 2$, 1998, p. 463-484.

[8] Ghidagia J-M., Kumbaro A., Le Coq G. « On the numerical solution to two fluid models via a cell centred finite volume method », Eur. J. b fluids, vol. 20, n 2, 1998, p. 463-484.

[9] Greenberg, J. M., Leroux A. Y. « A well-balanced scheme for the numerical processing of source terms in hyperbolic equations », SIAM J. Numer. Anal. , vol. 33, n 1, 1996, p. 1-16.

[10] Gosse, L. «A priori error estimate for a well-balanced scheme designed for inhomogeneous scalar conservation laws », C. R. Acad. Sci. Paris Sér. I Math. , vol. 327, n 5, (1998), 467-472.

[11] Harten, A., Lax, P.D., VAn Leer, B. « On Upstream Differencing and godunov-type Schemes for Hyperbolic Conservation Laws », SIAM Review, 25, 35-61, 1983.

[12] Hinch, E. J., « Perturbation methods », Cambridge University Press, Cambridge 1991.

[13] Masella, J-M., Faille, I., GallouËT, T., « On an approximate Godunov scheme », International Journal of Computational Fluid Dynamics, vol. 12, n ${ }^{\circ}$ 2, 1999, p. 133-149.

[14] LAHAYE R. T. « The prediction phase of phase distribution and separation phenomena using two-fluid models ", Boiling heat transfer, Elsevier Science, vol. 147, n 6, 2001, p. 841-867.

[15] Ransom, V. H., Hicks D. L., " Hyperbolic two-pressure models for two-phase flow », Journal of Computational Physics, vol. 75, $\mathrm{n}^{\circ}$ 2, 1988, p. 498-504.

[16] RoE, P. L. " Approximate Riemann solvers, parameter vectors, and difference schemes », Journal of Computational Physics, vol. 43, 1981, p. 357-372.

[17] Sahmim, S., Benkhaldoun, F., Alcrudo, F. « A finite volume solver based on matrix sign for non homogeneous systems », FVCA4, HPS, F. Benkhaldoun, D. Ouazar(Eds), S. Raghay, Proceedings of the fourth International Symposium on Finite Volumes for Complex Application, 2005, p. 471-482.

[18] S Aнmiм, S. «Un schéma aux volumes finis avec matrice signe pour les systèmes non homogènes », Ph.D. Thesis Université paris 13, 2005.

[19] Seguin, N. «Modélisation et simulation numérique des écoulements diphasiques », Ph.D. Université de Provence, 2002.

[20] Toumi, I., Kumbaro, A., « An approximate linearized Riemann solver for a two-fluid model », Journal of Computational Physics, vol. 124, n 2, 1996, p. 286-300. 

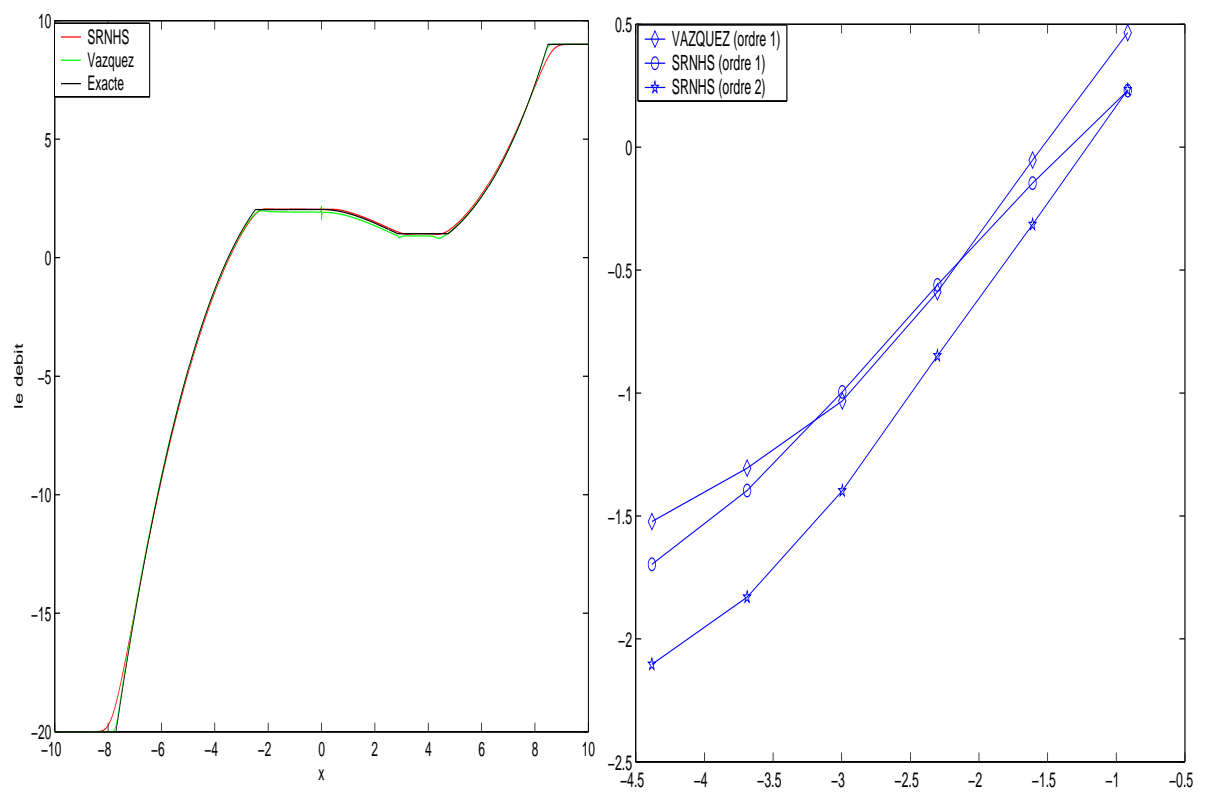

Figure 1. Equations de Saint-Venant (transition critique - ordre 1). Débit.

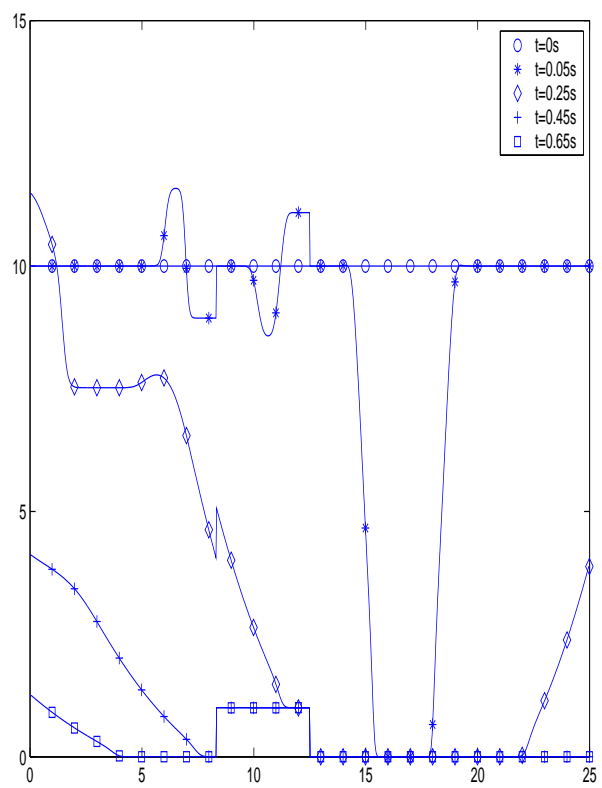

Figure 3. Surface libre d'un écoulement d'eau sur un obstacle, avec apparition de fond sec.
Figure 2. Transition critique. Courbe d'erreur sur la hauteur.

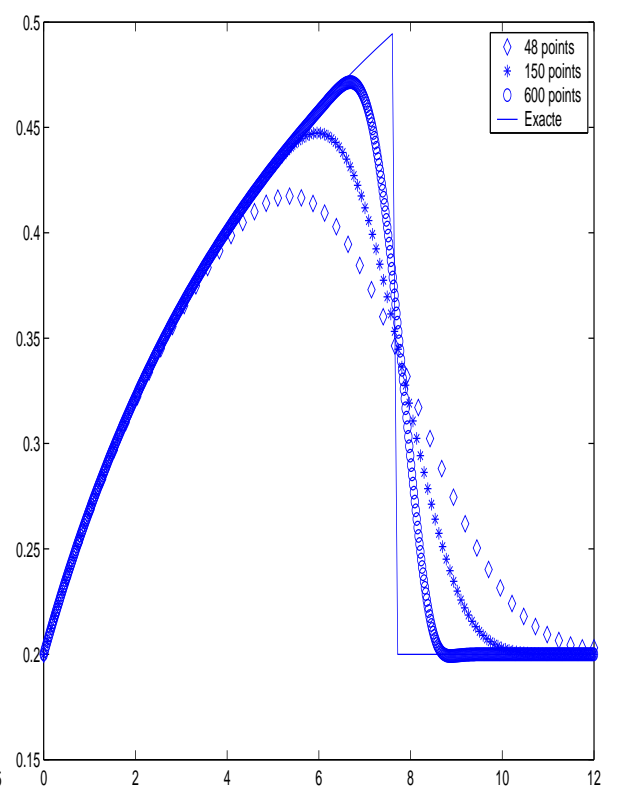

Figure 4. Ecoulement diphasique (Robinet de Ransom). Taux de vide de la vapeur à $t=0,6 s$. 